\title{
ACTUALIZACIÓN DE LA DOCENCIA UNIVERSITARIA A TRAVÉS DE LOS BLOGS EDUCATIVOS: SU APLICACIÓN A LOS ESTUDIOS DE TRABAJO SOCIAL

\begin{tabular}{l} 
UPDATE OF UNIVERSITY TEACHING THROUGH EDUCATIONAL \\
BLOGS: ITS APPLICATION TO SOCIAL WORK STUDIES \\
\hline
\end{tabular}

Enric Sigalat-Signes

Profesor del departamento de Trabajo Social y Servicios Sociales de la Universitat de València. Codirector de la Càtedra Participació, Govern Obert i Open Data de la Universitat de València. Departamento de Trabajo Social y Servicios Sociales. Universitat de València, Facultad de Ciencias Sociales, Valencia, (España). E-mail: enrique.sigalat@uv.es ORCID: https://orcid.org/0000-0001-8146-0532

Laura Llop-Medina

Graduada en Trabajo Social con mención en Interculturalidad, Cooperación Social y Exclusión Social. Master en Bienestar Social con especialidad en Investigación social. Personal Investigador en Formación. Universitat de València, Instituto Universitario de Políticas de Bienestar Social (Polibienestar), Valencia, (España). E-mail: laura.llop@uv.es ORCID: https://orcid.org/0000-0003-2084-4385

Lidia Bueno-Sánchez

Graduada en Trabajo Social con mención en Interculturalidad, Cooperación Social y Exclusión Social. Master en Bienestar Social con especialidad en Investigación social. Personal Investigador en Formación.Universitat de València, Instituto Universitario de Políticas de Bienestar Social (Polibienestar), Valencia, (España). E-mail: lidia.bueno@uv.es ORCID: https://orcid.org/0000-0002-0241-2717

Francisco Ródenas-Rigla

Director del departamento de Trabajo Social y Servicios Sociales e investigador senior del Instituto de Investigación Polibienestar de la Universitat de València. Departamento de Trabajo Social y Servicios Sociales. Universitat de València, Instituto Polibienestar, Valencia, (España).

E-mail: francisco.rodenas@uv.es ORCID: https://orcid.org/0000-0003-3264-4735

Recepción: 17/09/2021 Aceptación: 13/10/2021 Publicación: 29/12/2021

\section{Citación sugerida:}

Sigalat-Signes, E., Llop-Medina, L., Bueno-Sánchez, L., y Ródenas-Rigla, F. (2021). Actualización de la docencia universitaria a través de los blogs educativos: su aplicación a los estudios de Trabajo Social. 3C TIC. Cuadernos de desarrollo aplicados a las TIC, 10(4), 17-31. https://doi.org/10.17993/3ctic.2021.104.17-31 


\section{RESUMEN}

El uso de herramientas colaborativas de aprendizaje en la enseñanza universitaria es una oportunidad para actualizar la docencia. En los últimos años, los Blogs educativos han estado presentes como un facilitador del aprendizaje autónomo. En la asignatura "Investigación en Sistemas de Bienestar Social", del Grado de Trabajo Social de la Universitat de València (UV), se ha co-diseñado un blog grupal con el objetivo de fomentar la comunicación y el aprendizaje colaborativo entre el estudiantado y el profesorado. Para ello, se elaboró un cuestionario que se distribuyó entre el alumnado matriculado en la mencionada asignatura y que permitió conocer su experiencia previa y conocimiento de los blogs educativos, además de sus preferencias respecto a contenidos y potenciales usos de la herramienta. También se administró un cuestionario con carácter exploratorio entre el profesorado de la Facultad de Ciencias Sociales con el objetivo de conocer los potenciales usos de los blogs en la docencia universitaria. Los resultados muestran que el alumnado percibió el uso del blog como un instrumento favorecedor del aprendizaje colaborativo y participativo. Por su parte, el profesorado consideró la herramienta blog un facilitador de la práctica docente con múltiples utilidades, destacando especialmente la posibilidad de trabajar con el alumnado e intercambiar información. Como conclusión, la herramienta blog puede facilitar la coordinación docente y la comunicación horizontal con el estudiantado fomentando la participación activa del mismo y actualizando la docencia.

\section{PALABRAS CLAVE}

Herramientas colaborativas, Blog grupal, Actualización de la docencia, Co-creación, Enseñanza universitaria, Trabajo Social. 


\section{ABSTRACT}

The use of new collaborative learning tools at the university is an opportunity to update teaching. In recent years educational blogs have been present as a facilitator of autonomous learning. In the subject "Research in Social Welfare Systems" of the Degree in Social Work of the University of Valencia (UV), a group blog has been co-designed with the aim of promoting communication and collaborative learning between students and teachers. A questionnaire was prepared and distributed among the students enrolled in the aforementioned subject, allowing us to know their previous experience and knowledge of educational blogs, as well as their preferences regarding contents and potential uses of the tool. An exploratory questionnaire was also distributed among teachers of the Faculty of Social Sciences with the aim of knowing the potential uses of blogs in university teaching. The results show that the students perceived the use of the blog as a facilitator of collaborative and participatory learning. And teachers considered the blog tool a facilitator of teaching practice with multiple utilities highlighting, especially the possibility of working with students and exchanging information. In conclusion, the blog tool can facilitate teacher coordination and horizontal communication with students, encouraging their active participation and updating teaching.

\section{KEYWORDS}

Collaborative tools, Group blog, Teaching update, Co-creation, University teaching, Social Work. 


\section{INTRODUCCIÓN}

En el nuevo Espacio Europeo de Educación Superior (EES) los métodos de enseñanza han de potenciar la capacidad de aprendizaje autónomo por parte del estudiante, la resolución de problemas y el fomento del trabajo colaborativo. Las TIC en general y el uso de los blogs en particular, se han asentado en la última década en el contexto universitario (Duart y Lupiáñez, 2005; Santoveña, 2011; Marín, 2013; Ifinedo, 2018). Entre otras características cabe destacar que éstos propician la comunicación horizontal, presentan una alta versatilidad, cercanía y un nulo o bajo coste al tratarse de productos de acceso libre (Top, 2012; García, 2018). No obstante, de la misma manera, existen aspectos negativos referidos a otras experiencias menos positivas que tienen que ver esencialemente con el abandono y/o desatención del blog como herramienta educativa (González y García, 2011).

En la enseñanza universitaria española se han empleado blogs individuales, sin embargo, no suelen utilizarse blogs colectivos de aprendizaje. Uno de los principales requerimientos que aparece en la evaluación de las iniciativas con blogs individuales es lo beneficioso que resultaría el desarrollo de estos blogs de manera grupal. Responder a este requerimiento es una parte importante de la metodología del presente trabajo. Además, la co-creación en el aula es todavía una metodología poco empleada, por lo que desde este proyecto se ha impulsado. Esta metodología favorece el trabajo en equipo, las competencias sociales y coloca al estudiantado como una parte activa en su proceso de aprendizaje, incluso en momentos de inflexión como los vividos durante los últimos dos cursos académicos como consecuencia de la COVID-19. Con todo, hemos de considerar que la red es hoy por hoy un instrumento social que ayuda en la construcción del conocimiento y de las identidades, además de permitir la incorporación de las personas a la vida activa en la sociedad. De ahí que se haya convertido en un recurso, atreviéndonos a calificarlo de imprescindible para el crecimiento de los individuos y de las instituciones (Marín, 2013, p. 99).

En este artículoo se presenta una experiencia de innovación docente interdisciplinar. Con el objetivo de actualizar la docencia, a través del uso de las redes sociales para la construcción e intercambio de 
conocimiento, se ha continuado el trabajo iniciado en el curso 2019-2020 para codiseñar junto al alumnado un blog grupal didáctico de acceso libre denominado "Investiblog 2.0". El proyecto se puso en práctica en 4 grupos (A, B, C y G) de la asignatura "Investigación en Sistemas de Bienestar Social" del Grado en Trabajo Social de la Universitat de València (UV) durante el curso académico 2020/2021.

\section{METODOLOGÍA}

La experiencia de innovación docente que se presenta está basada en un estudio de caso de carácter exploratorio, que parte del análisis descriptivo de las respuestas sobre datos básicos y, en particular, en la aplicación de cuestionarios a los informantes considerados clave (alumnado de la asignatura y profesorado). Por ello, no se pretende concluir con el establecimiento de generalizaciones, sino más bien llegar a un enfoque analítico de la comprensión de la realidad objeto de análisis.

El proyecto que presentamos, eminentemente participativo, consideró para la co-creación de la citada herramienta, la opinión tanto del alumnado de la asignatura como del profesorado del Grado de Trabajo Social y otras titulaciones de la Facultad de Ciencias Sociales de la UV. A ambos colectivos, se les pasó un cuestionario mediante la utilización de la herramienta "Formularios de Google" (Google Docs). Al alumnado, con el objetivo de establecer si conocían el funcionamiento de un blog educativo, los tipos de contenidos que les gustaría compartir en la asignatura y los potenciales usos que realizarían de la herramienta, para posteriormente incluirlos en la misma. Al profesorado, con el objeto de conocer el uso que hacían de los blogs y establecer las posibilidades de estos como herramientas de comunicación, relación y colaboración entre docentes y alumnado. La participación en la creación del blog (Investiblog 2.0) y la respuesta a los cuestionarios de evaluación fue voluntaria, informando al alumnado de que dicha participación no tendría ninguna incidencia en sus calificaciones. 


\section{RESULTADOS}

En primer lugar, previamente al co-diseño del blog y para tratar de atender cualquiera de las propuestas de mejora que se pudieran detectar a la hora de implementar el mismo, se suministró en la primera semana del curso académico (febrero de 2021), un cuestionario de carácter voluntario a 4 grupos de la asignatura "Investigación en Sistemas de Bienestar Social" concretamente a los grupos A, B, C y G de tercer curso del Grado en Trabajo Social. Se obtuvieron un total de 140 respuestas. El cuestionario, formado por 11 preguntas, tenía como objetivo proporcionar información sobre el conocimiento del funcionamiento de un blog educativo, los tipos de contenidos que preferirían compartir y los potenciales usos que el alumnado realizaría de esta herramienta. La información resultante era importante para que el equipo docente pudiera reflejar e implementar posteriormente la misma en la nueva herramienta.

Por lo que atañe a los principales resultados, destacar que el 77\% del alumnado conoce el funcionamiento de los blogs. Así, un 73\% señala tener o haber tenido un blog. Un $62 \%$ del alumnado señala no haber usado nunca este tipo de herramientas en alguna asignatura a lo largo de su vida académica. El 38\% que ha utilizado un blog en alguna asignatura, señala mayoritariamente que ha sido en la educación secundariabachillerato $(75 \%)$. Un porcentaje menor, concretamente el 19,6\% del alumnado consultado, señala haber utilizado un blog en alguna asignatura en la Universidad. Por lo que se refiere a los contenidos que les gustaría compartir, la explicación visual de contenidos y la aportación de video y recursos virtuales, juntamente con documentos y enlaces con texto a los contenidos de la asignatura, son las opciones más señaladas, tal y como puede apreciarse en el siguiente gráfico: 


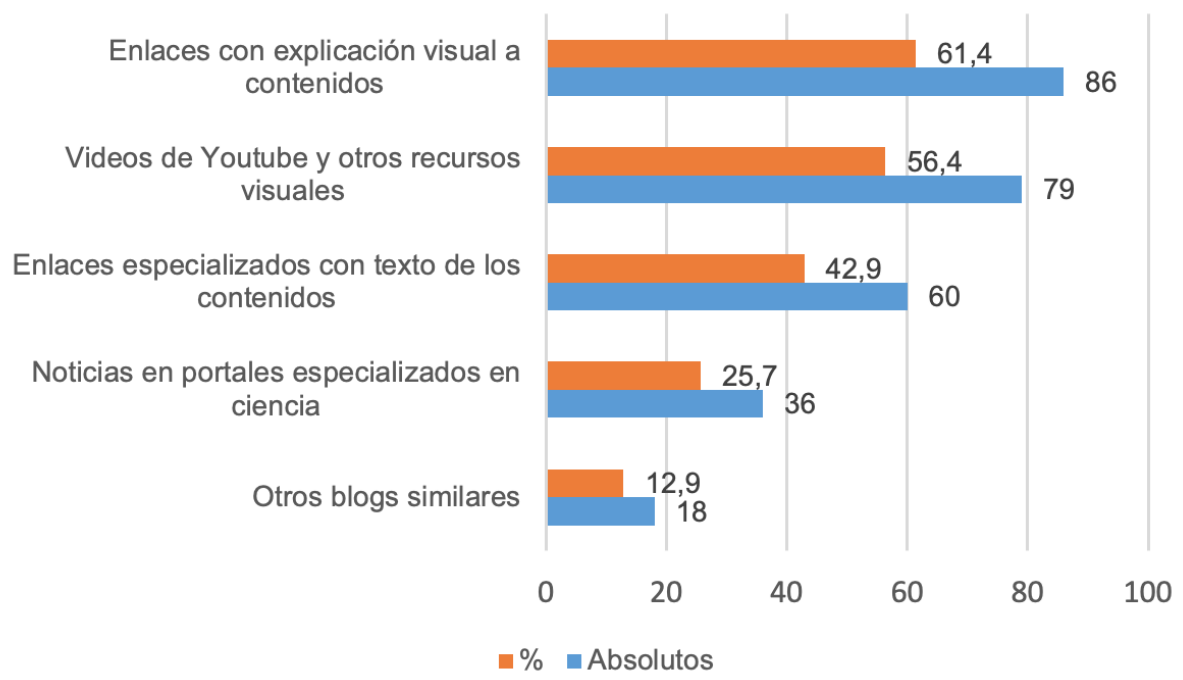

Gráfico 1. Contenidos a compartir en el blog por parte del alumnado. Multirespuesta.

Fuente: elaboración propia a partir del cuestionario para el alumnado.

El uso del blog como herramienta de trabajo en el aula tiene un impacto positivo en la percepción del alumnado, así pues el 89\% (9 de cada 10 por redondear) dice que considera adecuado la utilización de este tipo de herramientas en el sistema educativo. A un 8,6\% le resulta indiferente. Así mismo, cuando se les preguntaba por las ventajas del blog en relación a cuestiones pedagógicas y en las dinámicas propias del aula, la opinión mayoritaria del alumnado resulta altamente positiva para cada una de las cuestiones referenciadas (ver Tabla 1):

Tabla 1. Ítems planteados respecto a Investiblog 2.0.

\begin{tabular}{|c|c|c|c|c|c|}
\hline Ítems / \% & 1 & 2 & 3 & 4 & 5 \\
\hline $\begin{array}{l}\text { Considero adecuado el uso de un } \\
\text { blog en el sistema educativo }\end{array}$ & 0,7 & 1,4 & 8,6 & 40,7 & 48,6 \\
\hline $\begin{array}{l}\text { Considero que su utilización me } \\
\text { ayudará a mejorar el conocimiento } \\
\text { teórico-práctico de la asignatura }\end{array}$ & 0,7 & 2,9 & 10 & 45,7 & 40,7 \\
\hline
\end{tabular}




\begin{tabular}{|c|c|c|c|c|c|}
\hline $\begin{array}{l}\text { Me parece una herramienta } \\
\text { pedagógica muy pertinente a } \\
\text { incorporar en momentos actuales } \\
\text { como los de la covid-19 }\end{array}$ & 0,7 & 1,4 & 9,3 & 30 & 58,6 \\
\hline $\begin{array}{l}\text { Me parece una excelente } \\
\text { herramienta para el desarrollo } \\
\text { de dinámicas colaborativas y } \\
\text { participativas }\end{array}$ & 0,7 & 1,4 & 15,7 & 37,9 & 44,3 \\
\hline $\begin{array}{l}\text { Fomenta la interacción y el } \\
\text { intercambio y debate de ideas } \\
\text { entre el alumnado }\end{array}$ & 1,4 & 1,4 & 15,7 & 34,3 & 47,1 \\
\hline $\begin{array}{l}\text { Me parece interesante poder } \\
\text { acceder a la compilación de } \\
\text { trabajos, prácticas y exposiciones } \\
\text { de compañeros/as }\end{array}$ & 0,7 & & 7,9 & 30,7 & 60,7 \\
\hline
\end{tabular}

Escala del 1 al 5 (donde el 1 significa nada adecuado y el 5 significa muy adecuado).

Fuente: elaboración propia a partir del cuestionario para el alumnado.

Así mismo, el 61,4\% del alumnado, 6 de cada 10 alumnos/as, señalan que animarían al resto del profesorado de otras asignaturas a utilizar un blog como recurso educativo y 3 de cada 10 señalan que probablemente. El alumnado considera esta herramienta como un elemento facilitador de información y estudio compartido. Sobre todo, obtiene mayor relevancia en el periodo de confinamiento vivido este año por el estado de alarma derivado de la COVID-19. Las reuniones mantenidas por el profesorado de la asignatura, a lo largo del curso académico, con el alumnado de los grupos implicados en la implementación de esta herramienta, trasladan una retroalimentación altamente positiva respecto al uso del blog.

En segundo lugar, en el mes de mayo se suministró un cuestionario al profesorado. Este cuestionario tenía un carácter marcadamente exploratorio, y su objeto era conocer y entender mejor el uso y las posibilidades de los blogs como recurso didáctico en el sistema educativo universitario. Para ello se diseñó un cuestionario semiestructurado que estaba conformado por 11 preguntas, siendo dos de éstas de respuesta múltiple. El cuestionario fue respondido por 25 docentes de alguno de los siguiente títulos: 
Trabajo Social, Derecho, Relaciones Laborales, Magisterio, Doble Grado en Turismo y ADE, Sociología y Ciencias Políticas.

Destacar que el 86\% del profesorado encuestado, considera que puede ser útil un blog como herramienta educativa para su práctica docente. Un 16\% considera que probablemente puede ser una herramienta útil para el anterior cometido. No obstante, la gran mayoría (88\%) señala que no utiliza este tipo de herramientas en el día a día en sus clases. Únicamente un 12\% señalan utilizar esta herramienta en sus clases. En línea con lo anterior, un 36\% del profesorado encuestado señala que tiene pensado utilizar algún día un blog para sus clases y un 56\% que es probable. El blog como recurso didáctico es visto por parte de los y las docentes como una herramienta positiva con múltiples utilidades, tal y como se puede observar en el Gráfico 2.

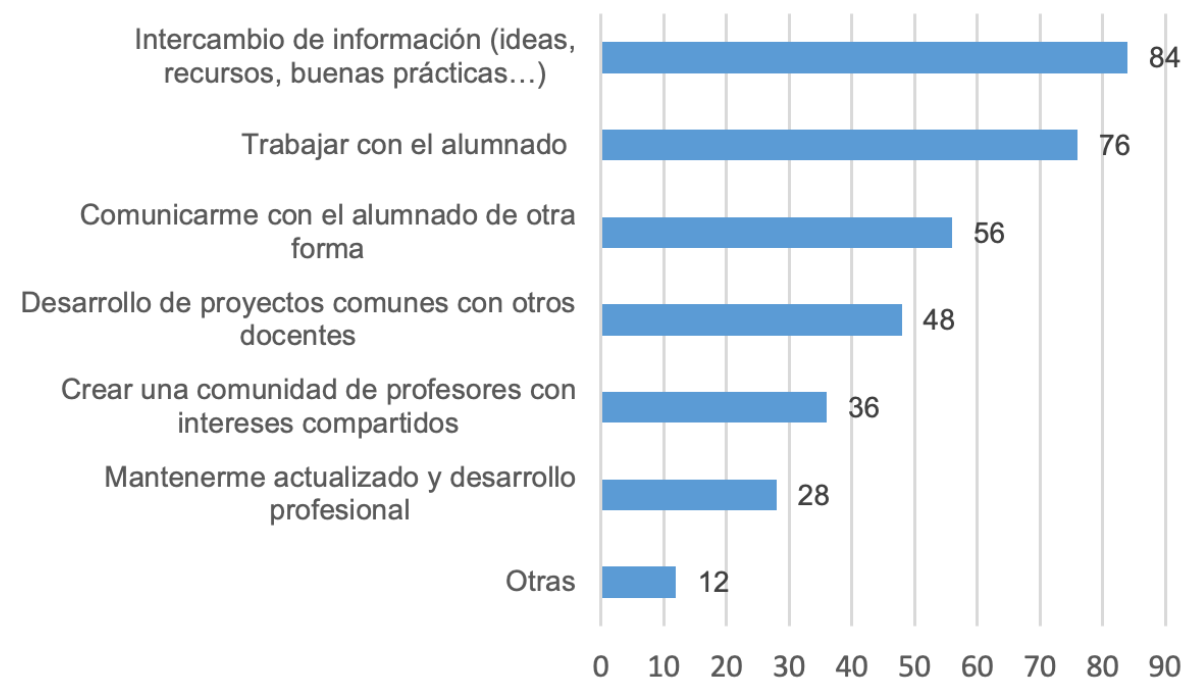

Gráfico 2. Utilidades de uso del blog para los docentes. Multirespuesta, En porcentaje.

Fuente: elaboración propia a partir del cuestionario para docentes.

Las opciones de intercambio de información y de poder trabajar con el alumnado, con un 84\% y un 76\% respectivamente, son las utilidades que destacan sobre el resto. En menor porcentaje, pero relativamente 
significativo, están también la posibilidad de comunicase con el alumnado de otra forma $(56 \%)$ y la de desarrollar proyectos comunes con otros docentes (48\%). Para los docentes, el uso más valorado en un blog es el de intercambio de información (4.72 puntos sobre 5 en una escala Likert donde 1 es la menor puntuación y el 5 es la máxima). Le siguen por igual, aunque en una puntuación menor, la posibilidad de tener una red de contactos y la integración de los contenidos con las redes sociales; ambas calificadas con 3.64 sobre la puntuación máxima de 5 puntos.

En la Tabla 2, se apuntan las principales dificultades manifestadas por los docentes a la hora de usar el blog en el aula. Como se puede observar, para los y las docentes la máxima dificultad por orden, estriba en la falta de ayuda y soporte técnico. Le siguen la inversión de tiempo que supone la iniciativa y la falta de formación.

Tabla 2. Principales dificultades en el uso del blog en el aula.

\begin{tabular}{|l|c|}
\hline Dificultades & Media \\
\hline Falta de formación técnica o tecnológica & 3.32 \\
\hline Poca convicción sobre su posible utilidad & 2.68 \\
\hline Inversión de tiempo de la iniciativa & 3.36 \\
\hline Falta de ayuda y soporte técnico & 3.64 \\
\hline Inseguridad en la utilización de estas herramientas & 2.96 \\
\hline
\end{tabular}

Escala de 1 (ninguna dificultad) a 5 (dificultad máxima).

Fuente: elaboración propia a partir del cuestionario para docentes.

En contraparte, entre las principales ventajas del uso del blog en el aula, los y las docentes señalan los beneficios de esta herramienta como recurso didáctico con puntuaciones en general altamente positivas, tal como se puede observar en la Tabla 3.

Tabla 3. Principales ventajas del uso del blog en el aula.

\begin{tabular}{|l|c|}
\hline Ventajas & Media \\
\hline $\begin{array}{l}\text { Todos y todas pueden ver el blog a cualquier hora y en } \\
\text { cualquier lugar }\end{array}$ & 4.36 \\
\hline
\end{tabular}




\begin{tabular}{|l|c|}
\hline Prologan la tarea educativa más allá del aula & 4.16 \\
\hline $\begin{array}{l}\text { Promueven el uso de internet y se gana en competencia } \\
\text { digital }\end{array}$ & 4,12 \\
$\begin{array}{l}\text { Son un complemento idóneo de aprendizaje significativo } \\
\text { y colaborativo }\end{array}$ & 4.08 \\
\hline $\begin{array}{l}\text { Promueven la interacción y comunicación en todos los } \\
\text { sentidos }\end{array}$ & 4.04 \\
\hline $\begin{array}{l}\text { Ayudan a fomentar la motivación del alumnado } \\
\text { Son herramientas útiles para el alumnado }\end{array}$ & 4 \\
\hline $\begin{array}{l}\text { Fomentan la motivación de los y las docentes } \\
\text { Es una herramienta social que facilita la socialización del } \\
\text { alumnado }\end{array}$ & 3.92 \\
\hline
\end{tabular}

Escala de 1 (ninguna ventaja) a 5 (mucha ventaja).

Fuente: elaboración propia elaboración propia a partir del cuestionario para docentes.

Finalmente, señalar que el grado de implicación de los estudiantes de los distintos grupos ha sido, en general, positivo. Uno de los mayores logros, ha sido que, a lo largo de la puesta en marcha del proyecto desarrollado, se ha conseguido involucrar al profesorado de 4 grupos de la asignatura. Ello ha permitido generar una estrategia común y facilitar materiales y contenidos significativos, en relación a la materia, para la consulta del alumnado en cualquier momento y lugar a través del blog.

Las competencias que ha desarrollado la práctica docente innovadora en el alumnado, que se trabajaron a través de "Investiblog 2.0", están lógicamente vinculadas al plan de estudios del Grado en Trabajo Social y fueron las siguientes:

- Capacidad para investigar y producir conocimiento sobre la realidad social, los problemas sociales y la disciplina de Trabajo Social.

- Capacidad para iniciar procesos de difusión científica y transferencia de resultados.

- Capacidad para diseñar, obtener financiación, gestionar y evaluar proyectos de investigación.

- Capacidad de recopilar e interpretar datos relevantes (dentro del área de estudio) para emitir juicios que incluyan una reflexión sobre temas relevantes de índole científica. 
En este sentido, se han generado dos tipos de productos con diferente grado de incidencia sobre el aprendizaje. Por un lado, se ha conseguido finalizar con éxito el proyecto abordado, generando evidencias de aprendizaje diversas: compartir contenido visual referente a la temática (videos YouTube) que no estaban en el curso anterior, enlace del blog en el aula para su acceso a cualquier alumno/a de los grupos de la asignatura implicados y aportaciones de trabajos como entradas al blog. Por otro lado, este proyecto ha permitido compartir contenidos prácticos en cuatros grupos de la citada asignatura del Grado de Trabajo Social. Efectivamente, nuestras prácticas de aula se están dirigiendo hacia aprendizajes más activos, donde el empleo de dispositivas y manuales de texto se combina con recursos digitales. $\mathrm{Y}$ donde el profesorado ha intentado introducir actividades motivadoras y diseñar de forma conjunta una herramienta, a partir de la opinión del alumnado, que ayude al desarrollo de las competencias profesionales.

\section{CONCLUSIONES}

Aunque somos conscientes que es posible mejorar más la coordinación docente, entre los miembros de la asignatura, y la colaboración entre docentes y alumnado, creemos que este tipo de proyectos facilita esta tarea. Consideramos que, a pesar de las dificultades logísticas para coordinarnos, y de los problemas técnicos sobrevenidos y del confinamiento debido a la COVID-19, hemos conseguido motivar a la mayoría del alumnado en el uso de los blogs educativos. No obstante, la debilidad del proyecto es la dependencia de ayuda y soporte técnico, así como la falta de formación tecnológica al respecto. Cuestiones también señaladas por los docentes consultados y aspectos éstos que se erigen como propuestas de mejora a resolver de cara a futuros cursos.

En este proyecto, se ha conseguido la participación activa, en todo el proceso de aprendizaje, del alumnado, introduciendo una nueva metodología innovadora y motivadora, fomentando el trabajo colaborativo entre iguales, como apuntan Top (2012), García (2018), o Martín (2009). Dado que la asignatura busca comprometer al estudiantado en la gestión del conocimiento y dotarlo de herramientas 
y habilidades para analizar problemas sociales, la implementación de esta metodología docente enriquecerá el contenido de la misma, estimulando la creatividad. Así mismo, se dota al profesorado de nuevas herramientas para la actualización de la docencia (Wise, 2005), sacándolo de su zona de confort e implicándolo en el desarrollo de nuevas metodologías docentes.

Los resultados del proyecto refuerzan el uso de los blogs en la esfera educativa, ya que es una práctica apropiada y útil para el desarrollo de un rol activo del estudiantado (y de los docentes), impulsando comunidades de aprendizaje (O’Donnell, 2006). Los Blogs educativos tienen un gran potencial como herramienta en el ámbito de la enseñanza. Éstos se pueden adaptar a cualquier disciplina y metodología docente. Así mismo, también pueden adaptarse a cualquier nivel educativo - de hecho, son un recurso didáctico utilizado en todos los niveles educativos-. En este trabajo hemos analizado las características y posibilidades del uso del blog y cómo este uso favorece el aprovechamiento de los procesos de enseñanzaaprendizaje de acuerdo con las necesidades de la sociedad de la información y comunicación. La estructura de los blogs los convierten en herramientas muy útiles para utilizar en la enseñanza, destacando tres usos principales: 1) Forman un sistema de almacenamientos de contenidos e información de interés para el alumnado de la asignatura; 2) se gestionan plenamente a través de internet por lo que, en cualquier momento y lugar, conectados a través de cualquier dispositivo móvil el alumnado puede hacer uso de ellos; y 3) el docente deja de ser un mero transmisor de conocimiento y se convierte más en un orientador y filtro de información de calidad a la que el alumnado puede tener acceso.

Finalmente, la única cuestión que se debe tenerse presente a la hora de implementar este tipo de herramientas es contar con una buena cobertura y ayuda técnica, para que los docentes se concentren en los materiales y contenidos. La puesta en marcha de este proyecto ha supuesto un verdadero reto para todo el alumnado implicado y para los docentes de la asignatura, pero la experiencia ha sido altamente positiva y tiene posibilidades de generalización al resto de asignaturas del Grado, así como a asignaturas de otros títulos. 


\section{AGRADECIMIENTOS}

Los autores y autoras quieren agradecer al Servei de Formació Permanent i Innovació Educativa de la Universitat de València, la financiación del proyecto dentro del "Programa de Innovación Docente, 2020" (referencia UV-SFPIE_PID-1354164).

\section{REFERENCIAS BIBLIOGRÁFICAS}

Duart, J. M., y Lupiáñez, F. (2005). Estrategias en la introducción y uso de las TIC en la universidad. RUSC. Universities and Knowledge Society Journal, 2(1). http://dx.doi.org/10.7238/rusc.v2i1.243

García, J. (2018). Razones para el uso de blogs por maestros en formación. In IN-RED 2018. IV Congreso Nacional de Innovación Educativa y Docencia en Red (pp. 16-22). https://dialnet.unirioja.es/ servlet/articulo? codigo $=7404142$

González, R., y García, F.E. (2011). Recursos eficaces para el aprendizaje en entornos virtuales en el Espacio Europeo de Educación Superior: análisis de los edublogs. ESE. Estudios sobre educación, 20, 161-180. https://revistas.unav.edu/index.php/estudios-sobre-educacion/article/view/4545

Ifinedo, P. (2018). Determinants of students; continuance intention to use blogs to learn: An empirical investigation. Behaviour \& Information Technology, 37(4), 381-392. https://doi.org/10.1080/014492 9X.2018.1436594

Marín, V. (2013). Los blogs al servicio de la educación inclusiva. Nuevas dimensiones culturales. Interaç̧ões, 9(23), 88-101. http://revistas.rcaap.pt/interaccoes/article/view/2821/2325

Martín, O. (2009). Educar en comunidad: promesas y realidades de la Web 2.0 para la innovación pedagógica. En Carneiro, R.; Toscano, J.C y Díaz, T. (coords.): Los desafios de las TIC para el cambio educativo. Madrid: colección Metas Educativas 2021, OEI- Fundación Santillana. 
O’Donnell, M. (2006). Blogging como práctica pedagógica: Artefacto y ecología. Educador de medios de Asia Pacifico, (17), 5-19.

Santoveña, S. (2011). Incidencia de los nuevos alfabetismos en la mejora de la calidad de la enseñanza: El caso de los blogs. Aula Abierta, 39(2), 59-68. https://redined.mecd.gob.es/xmlui/handle/11162/3992

Top, E. (2012). Blogging as a social medium in undergraduate courses: Sense of community best predictor of perceived learning. The Internet and Higher Education, 15(1), 24-28. https:/ / www.learntechlib. $\mathrm{org} / \mathrm{p} / 53731 /$

Wise, L. (2005). Blogs versus discussion forums in postgraduate online continuing medical education. En Blogtalk downunder conference (pp. 19-22). Sydney. 